\title{
PERFIL DE ÓBITOS NEONATAIS EM UMA REGIÃO DO ESTADO DA BAHIA
}

\author{
PROFILE OF NEONATAL DEATHS IN A REGION OF THE STATE OF BAHIA \\ Maísa Mônica Flores Martins ${ }^{1}$, Andresa Bacelar da Paixão ${ }^{2}$
}

Doutoranda em Saúde pública pelo Instituto de Saúde Coletiva da UFBA. Professora da Universidade Católica do Salvador, Faculdade de Enfermagem, Salvador, BA, Brasil, / Instituto de Saúde Coletiva da UFBA ${ }^{1}$

Enfermeira pela Universidade Católica do Salvador, Faculdade de Enfermagem, Salvador, Bahia, Brasil ${ }^{2}$

\begin{abstract}
This is an epidemiological study, of an ecological type, of a descriptive character, which aimed to analyze the profile of neonatal deaths in the health region of Salvador, Bahia, Brazil, from 2009 to 2018. The study had as units of analysis ten municipalities in the health region of Salvador, Bahia. Performed from secondary data, with data from the Mortality Information System (SIM) and the Live Birth Information System (SINASC), with the variables being analyzed; sex, age, color / race, age and education of the mother, type of delivery, duration of pregnancy, place of birth and coverage of Primary Health Care. 5,090 cases of neonatal deaths were recorded, of which 4,046 were early neonatal deaths and 1,044 late neonates. Among the most frequently analyzed characteristics of newborns, the following stand out: being male, prematurity with extremely low weight, and among maternal conditions; be young, aged between 20 and 29 years old, self-styled brown, having vaginal delivery. It is concluded that neonatal deaths are linked to the socioeconomic conditions of the mother and / or family, in addition to being related to the quality of care, which should be considered as an alert for the health system in the planning of public policies and in the execution of actions in health services. Bearing in mind that, Primary Health Care has an effective structure for the reduction of infant deaths.
\end{abstract}

Resumo

Trata-se de um estudo epidemiológico, do tipo ecológico, de caráter descritivo, o qual objetivou analisar o perfil dos óbitos neonatais da região de saúde de Salvador, Bahia, Brasil, no período de 2009 a 2018. O estudo teve como unidades de análise dez municípios da região de saúde de Salvador, Bahia. Realizado a partir de dados secundários, com dados do Sistema de Informações sobre Mortalidade (SIM) e do Sistema de Informações de Nascidos Vivos (SINASC), sendo analisado as variáveis; sexo, idade, cor/raça, idade e escolaridade da mãe, tipo de parto, duração da gestação, local de ocorrência de parto e cobertura da Atenção Primária à Saúde. Foram registrados 5.090 casos de óbitos neonatais, destes, 4.046 foram óbitos neonatais precoces e 1.044 neonatais tardios. Dentre as características analisadas dos recémnascidos, mais frequentes, destacam-se: ser do sexo masculino, prematuridade com extremo baixo peso, e dentre as condições maternas; ser jovens, com faixa etária entre 20 e 29 anos, autodenominadas pardas, tendo como via de parto vaginal. Conclui-se que os óbitos neonatais estão vinculados às condições socioeconômicas da mãe e/ou família, além de apresentar relação com a qualidade assistencial, o que deve ser considerado como um alerta para o sistema de saúde no planejamento de políticas públicas e na execução das ações nos serviços de saúde. Tendo em vista que, a Atenção Primaria a Saúde possui uma estrutura eficaz para a redução dos óbitos infantis.

Palavras-chave: Mortalidade infantil; Situação Socioeconômica; Atenção Primária em Saúde; Assistência à Saúde.
Keywords: Infant mortality; Socioeconomic situation; Primary Health Care; Health Assistance. 


\section{Introdução}

A mortalidade infantil é caracterizada pela ocorrência de óbitos que acometem crianças menores de um ano de idade, sendo considerada como um indicador para análise da situação de saúde $^{1}$. Este indicador é influenciado pela combinação de fatores biológicos, sociais, culturais e de falhas do sistema de saúde ${ }^{2}$.

O Brasil vem evoluindo no que se refere à diminuição do índice de mortalidade infantil, alcançando uma taxa de 15,9 mortes a cada mil nascidos vivos, segundo o Censo de $2010^{3}$. O monitoramento da mortalidade infantil é indispensável para o reconhecimento dos fatores de risco, bem como, a análise dos impactos sociais, econômicos, a qualidade e a cobertura dos serviços de saúde prestados à população ${ }^{4}$.

O principal componente e os maiores índices de mortalidade infantil são caracterizados como neonatal, e grande parte das mortes infantis acontecem nas primeiras 24 horas (25\%), indicando uma relação estreita com a atenção ao parto e o nascimento ${ }^{5}$. As taxas de mortalidade no primeiro dia de vida foram maiores em nascidos vivos com características desfavoráveis das mães, a exemplo de mães sem escolaridade, além de condições próprias do indivíduo como baixo peso ao nascer ${ }^{6}$.

Dentre os fatores que influenciam este indicador, o pré-natal é considerado como um importante fator de proteção para a morte ${ }^{7}$, sendo uma das principais ações programáticas realizadas nos serviços de atenção primária à saúde (APS). Este serviço é considerado como principal porta de entrada para o sistema de saúde composto por uma diversidade de serviços realizados de acordo com as necessidades de saúde dos usuários ${ }^{8}$. Algumas características desenvolvidas nos serviços de APS podem interferir positivamente no desfecho de uma gestação, como uma gravidez bem acompanhada pela equipe de saúde, e adesão ampla aos cuidados ao iniciar o pré-natal no momento certo, são ações que podem propiciar qualidade a saúde da gestante e do bebê, com o intuito de prover uma gestação e parto mais saudáveis ${ }^{7-9}$.

Atualmente, mesmo com os avanços na área de saúde, na expansão do acesso aos serviços de APS e aumento da cobertura do atendimento às mães durante a gestação, ainda existem intercorrências de óbitos neonatais, devido às intercorrências durante a gestação, como: infecção urinária, corioamnionite e sofrimento fetal $^{10}$. Além disso, é importante considerar as condições socioeconômicas das famílias e estruturais do sistema de saúde, como barreiras acesso à saúde, além da necessidade de garantir uma atenção à saúde de qualidade durante todo o ciclo gravídico-puerperal ${ }^{11}$.

Tendo em vista que, muitas causas de óbitos neonatais são consideradas preveníveis, com adequado acesso e garantia de assistência de qualidade no que se refere ao pré-natal, parto e puerpério, especialmente, por meio de diagnósticos e intervenções precoces. Dessa forma, identificar e monitorar as causas de óbito no enfoque da evitabilidade representam passos importantes, pois possibilitam a construção de indicadores sensíveis à qualidade da atenção à saúde, acionam mecanismos de investigação para explicação dos óbitos e desencadeiam ações para sua redução ${ }^{12}$.

Portanto, nesse contexto, este estudo tem como objetivo analisar o perfil dos óbitos neonatais da região de saúde de Salvador, Bahia, Brasil, no período de 2009 a 2018, a fim de identificar os possíveis fatores que estão associados aos óbitos neonatais, de modo a propor intervenção que possam repercutir nos indicadores de mortalidade infantil, além de subsidiar as práticas de saúde e a proposição de novas políticas públicas.

\section{Metodologia}

Trata-se de um estudo epidemiológico, do tipo ecológico, de caráter descritivo, a partir de dados secundários, que foram colhidos através do Departamento de Informática do SUS (DATASUS).

Tendo como unidade de análise a região de saúde de Salvador, Bahia, composta por dez municípios, a saber: Candeias, Itaparica, Lauro de Freitas, Madre de Deus, Salvador, Santo Amaro, São Francisco do Conde, São Sebastião do Passé, Saubara e Vera Cruz, com um total estimado de 3.400.621 habitantes ${ }^{13}$.

Salvador é o município referência da região de saúde, com população estimada em 2.872.347 habitantes no ano de $2019^{13}$. É a terceira cidade mais populosa do país e comanda a sexta região metropolitana brasileira em termos demográficos $^{14}$. Em 2018, o salário médio mensal era de 3,5 salários mínimos. A proporção de pessoas ocupadas em relação à população total era de $29,7 \%$. Considerando rendimentos mensais de até meio salário mínimo por pessoa, sendo este contingente de $36,8 \%$ da população nestas condições. Em 2010, a taxa de escolarização, era de 6 a 14 anos de idade, 
possuía um PIB per capita $\mathrm{R} \$ 21.231,48$, e Índice de Desenvolvimento Humano Municipal de $0,759^{13}$.

A população do estudo foi composta por neonatos entre 0 a 27 dias de vida. Para observar o comportamento na variação de ocorrências, o estudo foi realizado com os óbitos registrados no período de 2009 a 2018 de mães residentes na região de saúde de Salvador, Bahia. Utilizou-se como fonte de dados, o Sistema de Informação sobre Mortalidade (SIM) e no Sistema de Informação sobre Nascidos Vivos (SINASC), e dados da cobertura da atenção básica disponíveis no e-gestor $A B^{15}$.

Foram utilizados para análise do estudo as variáveis: sexo, idade, cor/raça, idade e escolaridade da mãe, tipo de parto, duração da gestação, local de ocorrência de parto e cobertura da Atenção Primária à Saúde. O plano de análise do estudo, consiste no cálculo da frequência relativa das características demográficas, socioeconômicas e de assistência materna e da criança, o coeficiente de mortalidade infantil segundo o município de residência, que para o cálculo do indicador considerou a soma dos óbitos ocorridos nos períodos neonatal precoce $(0-6$ dias de vida), neonatal tardio (7 - 27 dias) e pósneonatal (28 - 364 dias) dividido pelo número de nascidos vivos $X$ 1000. Além do cálculo do coeficiente mortalidade neonatal segundo a região de saúde, nesta análise estratificou os casos precoces, que consistem no número de óbitos de neonatos de 0-6 dias de vida, dividido por o número de nascidos vivos, multiplicado por 1000, e, tardios, número de óbitos de neonatos de 7 - 27 dias de vida, dividido por o número de nascidos vivos, multiplicado por 1000 . Além do cálculo da média da cobertura da atenção básica para a região de saúde de Salvador, Bahia, sendo considerado a soma das coberturas de cada mês do ano dividido por 12 . Este cálculo foi realizado para cada ano do estudo, para os dados da cobertura da atenção básica e a Estratégia de Saúde da Família.

A inserção e a organização dos dados foram realizadas em Planilha Eletrônica do Microsoft Excel for Windows 2013 e apresentados em forma de tabelas e gráficos, inicialmente, para explanação dos dados, contendo informações das variáveis utilizadas nesse estudo.
Não foi necessário a submissão ao comitê de ética uma vez que os dados são de origem secundária.

\section{Resultados}

Na região de saúde de Salvador, no período de 2009 a 2018, foram confirmados 5.090 casos de óbitos neonatais. Quando analisados os dados por condições sociodemográficas, do perfil materno, observa-se que, existe uma grande concentração dos óbitos, de mães com idade entre 20 a 29 anos, o que corresponde a uma soma de quase $43 \%$ dos óbitos. Um outro grupo etário que chama a atenção é o de mães adolescentes com 15 a 19 anos que apresentou $15 \%$ da frequência de óbitos neonatais para o período estudado (Tabela 1). Para a variável raça/cor das mães observou uma maior concentração das autodenominadas pardas $(55,8 \%)$, e cerca de $32 \%$ foram consideradas ignorados (Tabela 1).

Com relação a escolaridade da mãe, para a região em estudo, os casos se concentram entre 8 a 11 anos $(36,6 \%)$ de estudos, tendo seu maior percentual em 2018 (47,3\%), seguido de um total de $17 \%$ das mães que tinham de 4 a 7 anos de estudos. Uma categoria que chama a atenção é a de ignorado com cerca de quase $28 \%$ total dos casos, para a variável escolaridade materna (Tabela 1).

Observa-se para a variável tipo de parto que cerca de $60 \%$ dos partos foram classificados com vaginais e $35,1 \%$ cesarianas. A disposição dos dados demonstrou um crescimento nos últimos anos para os casos de óbitos neonatais para os partos por via cesárea sendo registrado em 2009 (28\%) e em 2018 chegaram a aproximadamente $42 \%$ (Tabela 1). 


\begin{tabular}{|c|c|c|c|c|c|c|c|c|c|c|c|}
\hline IDADE DA MÃE & 2009 & 2010 & 2011 & 2012 & 2013 & 2014 & 2015 & 2016 & 2017 & 2018 & Total \\
\hline 10 a 14 anos & 1,4 & 1,3 & 0,9 & 1,0 & 1,7 & 2,6 & 1,1 & 1,8 & 1,1 & 0,2 & 1,3 \\
\hline 15 a 19 anos & 13,3 & 16,0 & 17,0 & 16,9 & 14,6 & 14,9 & 15,4 & 14,2 & 12,5 & 14,2 & 15,0 \\
\hline 20 a 24 anos & 24,3 & 24,9 & 20,6 & 19,1 & 22,3 & 20,4 & 21,1 & 24,0 & 21,0 & 20,4 & 21,8 \\
\hline 25 a 29 anos & 20,4 & 21,7 & 22,0 & 23,0 & 21,4 & 22,2 & 20,0 & 16,5 & 21,6 & 19,1 & 20,8 \\
\hline 30 a 34 anos & 12,5 & 15,1 & 15,0 & 16,5 & 17,1 & 15,9 & 20,9 & 21,9 & 23,3 & 20,0 & 17,7 \\
\hline 35 a 39 anos & 9,2 & 6,7 & 9,3 & 10,1 & 9,4 & 14,5 & 11,4 & 15,4 & 13,0 & 16,8 & 11,5 \\
\hline 40 a 44 anos & 3,3 & 2,4 & 2,1 & 3,3 & 3,0 & 3,7 & 3,8 & 3,0 & 2,8 & 4,2 & 3,1 \\
\hline 45 a 49 anos & 0,0 & 0,4 & 0,0 & 0,0 & 0,5 & 0,2 & 0,2 & 0,0 & 0,2 & 0,2 & 0,2 \\
\hline 50 a 54 anos & 0,0 & 0,0 & 0,0 & 0,0 & 0,0 & 0,0 & 0,2 & 0,0 & 0,0 & 0,4 & 0,1 \\
\hline Idade ignorada & 15,7 & 11,5 & 13,1 & 10,1 & 9,9 & 5,5 & 5,9 & 3,3 & 4,5 & 4,5 & 8,5 \\
\hline \multicolumn{12}{|c|}{ ESCOLARIDADE DA MÃE } \\
\hline Nenhuma & 7,2 & 6,3 & 4,5 & 4,1 & 3,5 & 2,9 & 1,9 & 2,8 & 2,8 & 3,2 & 4,0 \\
\hline 1 a 3 anos & 3,5 & 2,8 & 3,6 & 3,9 & 5,6 & 3,1 & 5,9 & 5,3 & 3,2 & 1,3 & 3,9 \\
\hline 4 a 7 anos & 14,7 & 14,1 & 15,3 & 17,5 & 13,9 & 20,0 & 17,5 & 20,3 & 18,6 & 18,7 & 17,0 \\
\hline 8 a 11 anos & 24,5 & 32,5 & 33,7 & 36,2 & 33,6 & 39,1 & 39,2 & 41,3 & 41,0 & 47,3 & 36,6 \\
\hline 12 anos e mais & 8,2 & 11,0 & 6,6 & 9,3 & 9,8 & 10,4 & 13,3 & 12,6 & 14,9 & 14,4 & 10,9 \\
\hline Ignorado & 41,9 & 33,3 & 36,2 & 29,0 & 33,6 & 24,4 & 22,2 & 17,7 & 19,4 & 15,1 & 27,6 \\
\hline \multicolumn{12}{|l|}{ RAÇA/COR } \\
\hline Branca & 6,8 & 8,2 & 9,1 & 8,2 & 6,6 & 7,5 & 5,1 & 6,9 & 5,4 & 5,9 & 7,0 \\
\hline Preta & 3,3 & 1,9 & 5,1 & 2,9 & 4,4 & 4,9 & 3,6 & 5,9 & 9,3 & 13,4 & 5,3 \\
\hline Amarela & 0,0 & 0,0 & 0,2 & 0,0 & 0,2 & 0,2 & 0,0 & 0,0 & 0,2 & 0,0 & 0,1 \\
\hline Parda & 52,1 & 56,9 & 52,3 & 50,4 & 49,8 & 55,0 & 61,8 & 67,5 & 62,2 & 51,4 & 55,8 \\
\hline Ignorado & 37,8 & 33,1 & 33,3 & 38,5 & 39,0 & 32,4 & 29,5 & 19,7 & 22,9 & 29,3 & 31,8 \\
\hline \multicolumn{12}{|l|}{ TIPO DE PARTO } \\
\hline Vaginal & 66,1 & 61,7 & 59,1 & 59,3 & 60,3 & 62,3 & 58,0 & 57,1 & 57,2 & 56,3 & 59,8 \\
\hline \multirow{2}{*}{$\begin{array}{l}\text { Cesário } \\
\text { Ignorado }\end{array}$} & 28,0 & 32,7 & 32,8 & 34,2 & 32,2 & 33,4 & 38,4 & 40,0 & 40,6 & 40,8 & 35,1 \\
\hline & 5,9 & 5,6 & 8,1 & 6,4 & 7,5 & 4,3 & 3,6 & 3,0 & 2,2 & 3,0 & 5,0 \\
\hline
\end{tabular}


Quando analisados os dados por condições sociodemográficas dos óbitos infantis neonatais, segundo as informações da criança, observou-se que houve predominância do sexo masculino em todos os anos, sendo 2.729 casos em números absolutos, o que corresponde a $53,6 \%$ dos casos (Tabela 2).

De acordo com as características individuais do neonato, foi possível observar que a distribuição de casos se concentra em neonatos com extremo baixo peso (38,8\%), ou seja, os que apresentaram de 500 a 999g, que, sendo que no primeiro ano de estudo, apresentaram 42,1\%. Pelos resultados apresentados foi possível observar que esta variável apresenta informações bastante relevantes para o problema estudado, em que, as maiores concentrações dos óbitos foram em recém-nascidos que apresentaram em sua totalidade, piores condições do peso, com peso menor de $500 \mathrm{~g}(12,8 \%)$, de 1000 a $1499 \mathrm{~g}(12,9 \%)$ e 1500 a $2499 \mathrm{~g}(14,1 \%)$. Quanto a idade/duração da gestação, houve maior frequência de óbitos em recém-nascidos prematuros, de 22 a 27 semanas de gestação (32,7\%) (Tabela 2$)$. No que se refere ao local de ocorrência do parto, houve predominância em partos ocorridos em Hospital $(97,7 \%)$, e no domicílio apenas 0,5\% (Tabela 2 ).

Tabela 2: Distribuição dos fatores associados à mortalidade infantil neonatal, de acordo com as características demográficas, e assistências da criança. Bahia, 2009 a 2018.

\begin{tabular}{lccccccccccc}
\hline \multicolumn{1}{c}{ SEXO } & $\mathbf{2 0 0 9}$ & $\mathbf{2 0 1 0}$ & $\mathbf{2 0 1 1}$ & $\mathbf{2 0 1 2}$ & $\mathbf{2 0 1 3}$ & $\mathbf{2 0 1 4}$ & $\mathbf{2 0 1 5}$ & $\mathbf{2 0 1 6}$ & $\mathbf{2 0 1 7}$ & $\mathbf{2 0 1 8}$ & Total \\
\hline Masculino & 55,2 & 55,8 & 51,5 & 51,0 & 53,7 & 50,5 & 57,4 & 54,1 & 52,5 & 54,8 & 53,6 \\
Feminino & 42,7 & 42,0 & 47,5 & 46,1 & 44,4 & 47,3 & 42,0 & 43,1 & 44,5 & 42,5 & 44,2 \\
Ignorado & 2,2 & 2,2 & 0,9 & 2,9 & 1,9 & 2,2 & 0,6 & 2,8 & 3,0 & 2,8 & 2,1 \\
PESO AO NASCER (g) & & & & & & & & & & & \\
Menos de 500g & 8,4 & 10,4 & 9,8 & 14,4 & 12,2 & 15,7 & 13,5 & 12,8 & 12,3 & 18,7 & 12,8 \\
500 a 999g & 42,1 & 40,5 & 38,6 & 37,4 & 39,9 & 38,5 & 35,9 & 36,2 & 42,1 & 36,5 & 38,8 \\
1000 a 1499 g & 12,9 & 11,0 & 11,6 & 12,6 & 12,4 & 13,9 & 14,6 & 14,6 & 13,0 & 13,0 & 12,9 \\
1500 a 2499 g & 14,7 & 14,3 & 16,9 & 12,8 & 13,4 & 10,8 & 16,0 & 17,1 & 12,7 & 12,5 & 14,1 \\
2500 a 2999 g & 5,5 & 8,2 & 9,1 & 7,2 & 6,3 & 5,5 & 6,1 & 7,5 & 7,6 & 6,2 & 6,9 \\
3000 a 3999 g & 9,0 & 7,2 & 5,5 & 7,2 & 8,9 & 11,0 & 9,3 & 9,1 & 8,2 & 8,9 & 8,4 \\
4000g e mais & 1,2 & 2,0 & 1,1 & 1,8 & 1,0 & 0,8 & 1,3 & 0,8 & 1,1 & 0,8 & 1,2 \\
Ignorado & 6,3 & 6,3 & 7,4 & 6,6 & 5,9 & 3,7 & 3,4 & 2,0 & 3,0 & 3,4 & 4,9
\end{tabular}

\section{DURAÇÃO DA GESTAÇÃO}

Menos de 22 semanas

22 a 27 semanas

28 a 31 semanas

32 a 36 semanas

37 a 41 semanas

42 semanas e mais

Ignorado

\section{LOCAL DE OCORRÊNCIA}

Hospital

$\begin{array}{ccccccccccc}8,6 & 9,3 & 13,8 & 18,5 & 16,6 & 20,0 & 12,9 & 10,0 & 11,9 & 12,1 & 13,4 \\ 38,0 & 35,1 & 26,5 & 25,9 & 26,8 & 32,6 & 31,9 & 34,6 & 38,2 & 38,6 & 32,7 \\ 18,2 & 18,2 & 15,2 & 17,1 & 15,3 & 11,8 & 17,3 & 16,9 & 17,7 & 15,3 & 16,3 \\ 13,3 & 15,4 & 12,5 & 10,7 & 13,1 & 10,8 & 16,0 & 15,7 & 11,7 & 12,5 & 13,2 \\ 15,3 & 15,6 & 14,2 & 13,0 & 12,4 & 15,1 & 14,6 & 15,7 & 14,3 & 15,7 & 14,6 \\ 1,0 & 0,9 & 0,4 & 0,4 & 0,5 & 0,2 & 0,0 & 0,2 & 0,2 & 0,2 & 0,4 \\ 5,7 & 5,4 & 17,4 & 14,4 & 15,3 & 9,4 & 7,4 & 6,7 & 6,0 & 5,5 & 9,5\end{array}$

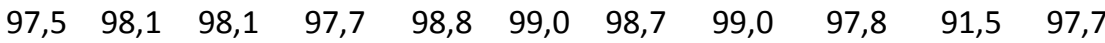


Continuação.

Outro estabelecimento

\begin{tabular}{lccccccccccc} 
de saúde & 0,8 & 1,1 & 0,9 & 1,8 & 0,3 & 0,6 & 0,6 & 0,4 & 1,5 & 8,1 & 1,6 \\
Domicílio & 1,0 & 0,4 & 0,4 & 0,6 & 0,7 & 0,2 & 0,6 & 0,4 & 0,2 & 0,2 & 0,5 \\
Via pública & 0,4 & 0,2 & 0,2 & 0,0 & 0,0 & 0,0 & 0,0 & 0,0 & 0,2 & 0,0 & 0,1 \\
Outros & 0,4 & 0,2 & 0,2 & 0,0 & 0,2 & 0,2 & 0,0 & 0,2 & 0,2 & 0,0 & 0,2 \\
Ignorado & 0,0 & 0,0 & 0,2 & 0,0 & 0,0 & 0,0 & 0,0 & 0,0 & 0,0 & 0,2 & 0,0 \\
\hline
\end{tabular}

Fonte: SIM/DATASUS

Observando os 10 municípios que compõem a região de saúde de Salvador é possível observar enorme variações entre eles e no período estudado. Dentre os municípios com coeficientes de mortalidade infantil muito elevadas destacamse Itaparica, no qual suas maiores taxas ocorreram em 2014 (32,39 para cada 1.000 nascidos vivos), 2012 com (29,2 para cada 1.000 nascidos vivos) e 2017 (25,75 para cada 1.000 nascidos vivos), seguido do município de Vera Cruz, onde apresentou o maior indicador em 2013 com (32,26 óbitos para cada 1000 nascidos vivos). No primeiro ano do estudo é possível observar que o município de Saubara foi o que apresentou maior taxa de mortalidade infantil com 30,49 óbitos para cada 1.000 nascidos vivos, entretanto nos anos seguintes há uma queda considerável, em 2010 apresentou 13,70 óbitos para cada 1000 nascidos vivos (Figura 1). Ainda com relação a figura 1 , apesar do município de Salvador ser o mais populoso da região de Saúde em estudo, e com um total de 363.149 nascidos vivos, quando se trata do coeficiente de mortalidade infantil, Salvador apresenta índices menores comparado a outros municípios da região de saúde, com um coeficiente médio de 11,7 óbitos para cada 1.000 nascidos vivos.

Figura 1: Taxa de mortalidade infantil neonatal ( 0 a 27 dias de vida) segundo os municípios da região de saúde de Salvador. Bahia, 2009 a 2018

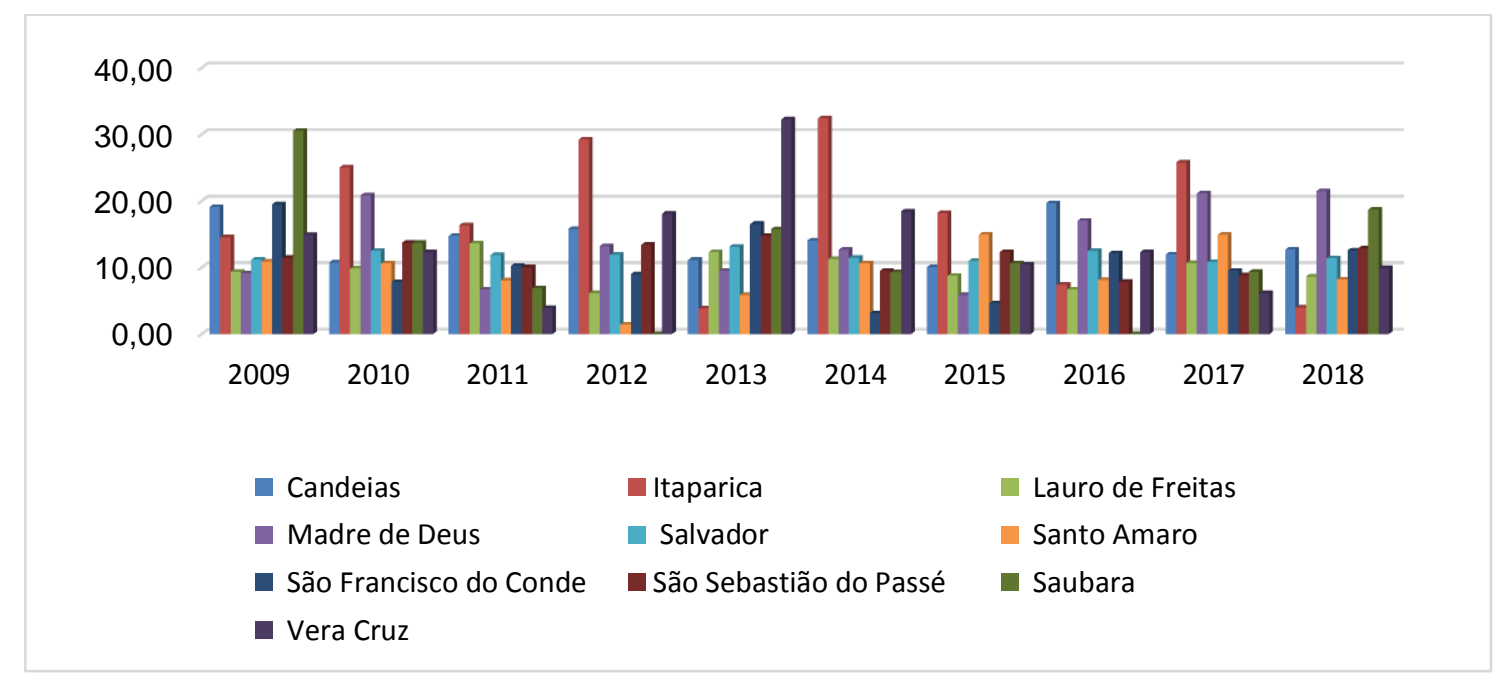

Fonte: SIM/SINASC/DATASUS 
Durante o período analisado foram registrados, por meio do SIM, 5.090 óbitos neonatais, destes, 4.046 foram óbitos neonatais precoces e 1.044 neonatais tardios (Figura 2). O coeficiente de mortalidade neonatal precoce manteve-se elevado e oscilante, principalmente nos anos de 2010, 2013 e 2016, foram os anos que apresentaram as maiores taxas de 10,11; 10,24; e 9,60 para cada 1.000 nascidos vivos, respectivamente (Figura 2). Enquanto que 0 coeficiente de mortalidade neonatal tardio apresentou-se com menores índices, comparado ao coeficiente de mortalidade neonatal precoce, no qual o maior coeficiente observado foi de 2,85 para cada 1.000 nascidos vivos em 2013, e a menor 1, 91 para cada 1.000 nascidos vivos, em 2018 (Figura 2).

Figura 2: Taxa de mortalidade infantil neonatal segundo faixa etária. Bahia, 2009 a 2018.

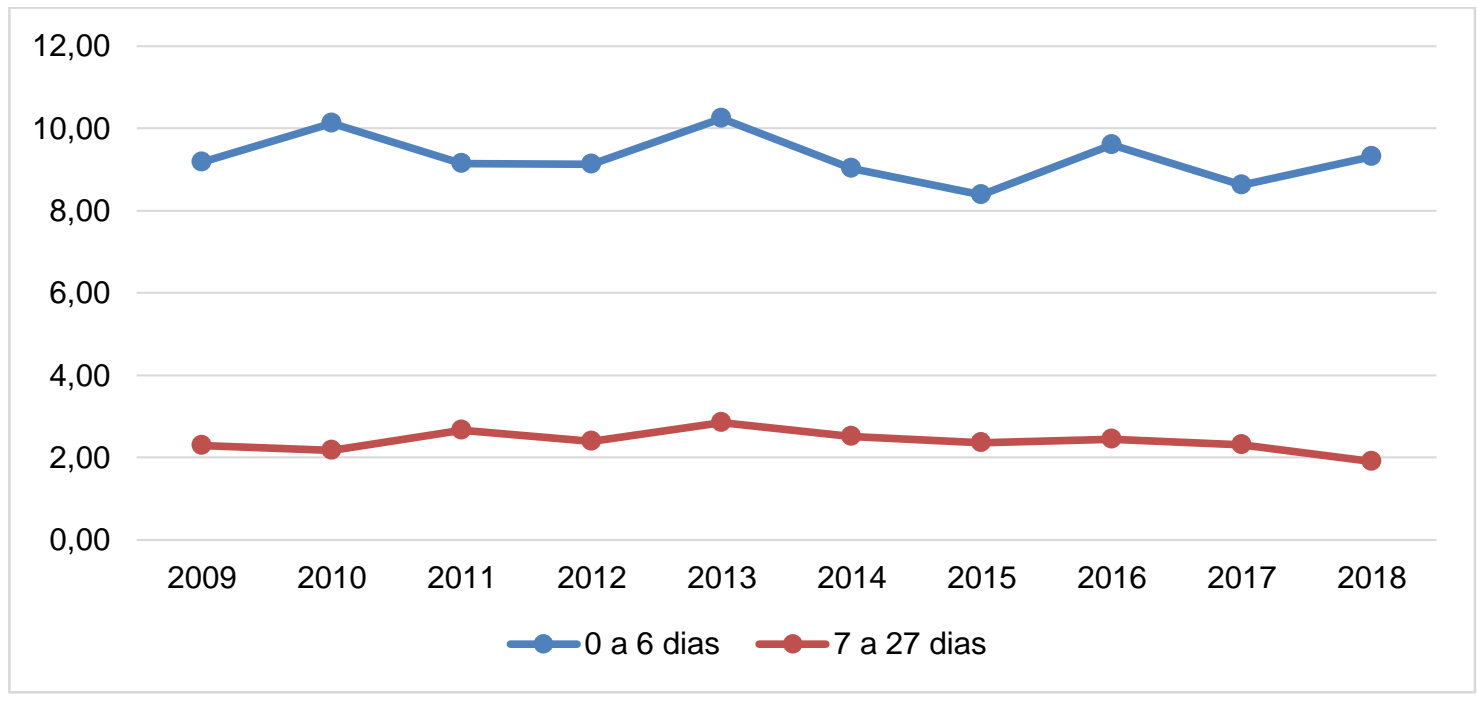

Fonte: SIM/SINASC/DATASUS 
De acordo com os dados de cobertura média da atenção básica há um comportamento de crescimento durante o período estudado, a Estratégia e Saúde da Família (ESF), permanece maior quando comparado a Atenção Básica (AB) em todos os anos. A cobertura da ESF mantevese elevada, principalmente, nos anos de 2016, 2017 e 2018, constando 43,81\%, 44,01\% e $44,79 \%$ de cobertura, respectivamente, sua menor taxa apresentou 33,33\% em 2009. Enquanto, que a cobertura da $A B$, demonstrou indicadores muito menores, sendo $17,51 \% \mathrm{em}$ 2009 a menor cobertura, e a maior $34,91 \%$ em 2016 (Figura 3).

Figura 3: Cobertura da Atenção Básica na região de saúde de Salvador, Bahia, 2009 a 2018.

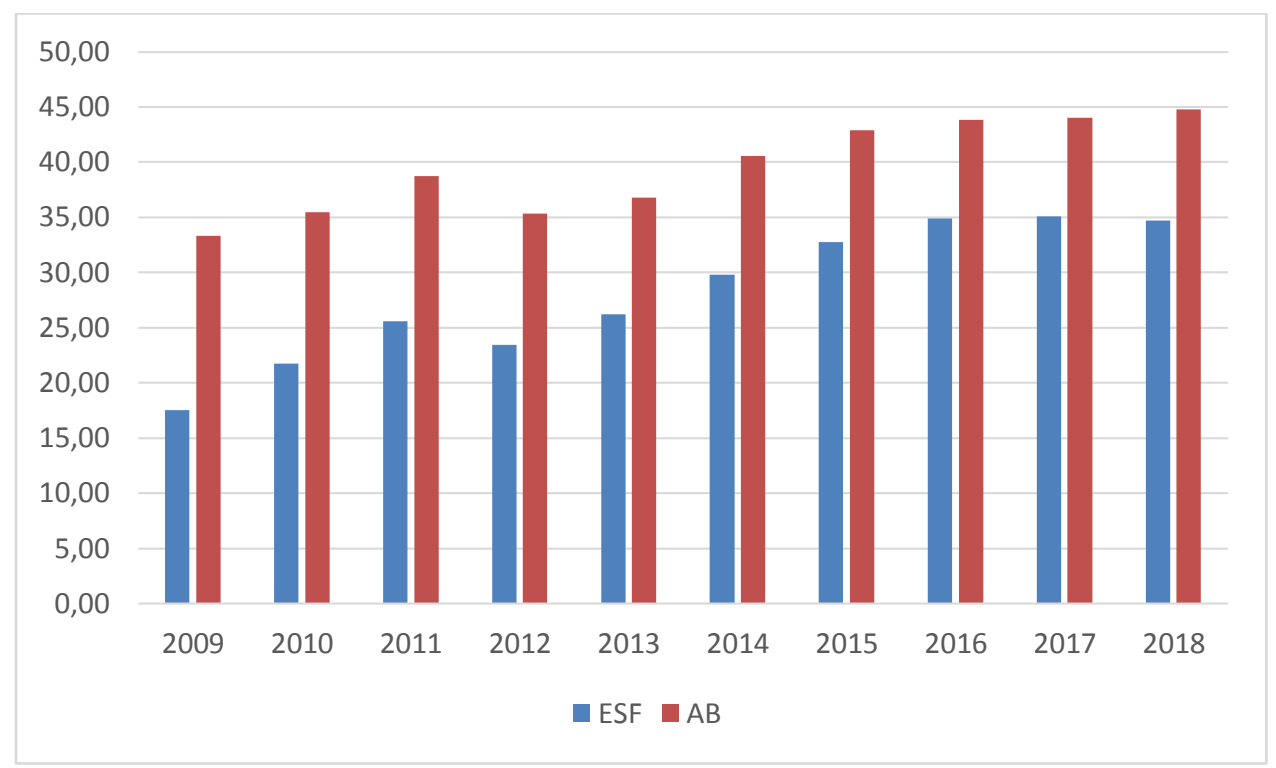

Fonte: e-Gestor/SUS 
A mortalidade neonatal é responsável por quase $70 \%$ das mortes no primeiro ano de vida, e o cuidado adequado ao recém-nascido tem sido um dos desafios para reduzir os índices de mortalidade infantil em nosso País ${ }^{16}$.

Um estudo publicado em 2016, observou que nos dias atuais, a mortalidade neonatal ( 0 a 27 dias de vida) tem a maior representação em termos proporcionais na mortalidade infantil no Brasil, representando até $70 \%$ do óbito infantil em todas as regiões do país ${ }^{17}$.

Neste presente estudo, constata-se que, sete fatores estão associados aos óbitos infantis, destes, três são de características infantis que foram identificados como: ser do sexo masculino, prematuridade, podendo ter associação ao extremo baixo peso, e, os demais fatores relacionados as condições maternas, dentre eles destaca-se; jovens, com faixa etária entre 20 e 29 anos, autodenominadas pardas, com 8 a 11 anos de estudos, tendo como via de parto vaginal.

Segundo o estudo, realizado em Caxias do Sul, por Araújo e Cols $^{18}$ (2000), em consonância com os resultados encontrados, os recém-nascidos que foram a óbito na primeira semana de vida eram, na sua maioria, do sexo masculino (64,3\%), e apresentavam peso baixo ao nascer. Ressalta ainda que, os $\mathrm{RN}$ de baixo peso apresentaram um risco de morrer na primeira semana de vida, com seis vezes maior do que os recém-nascidos com peso de nascimento igual ou maior do que $2.500 \mathrm{~g}$.

Segundo a literatura, o motivo de menor ocorrência de mortalidade do sexo feminino, é devido ao amadurecimento do pulmão fetal, que ocorre de forma mais antecipada no sexo feminino, o que reduz o risco de complicações respiratórias, que se associa entre as causas de óbitos no período neonatal ${ }^{18}$.

Um outro fator que se apresentou como causa importante para os óbitos neonatais no presente estudo foi a prematuridade, que está diretamente ligada ao extremo baixo peso. Esta característica é possível ser verificada em outras publicações científicas, como no estudo realizado na Região Sul do Brasil, mostrou que 93,1\% dos óbitos eram de prematuros ${ }^{19}$. Sendo a prematuridade considerada a principal causa de óbito neonatal em todas as regiões do país ${ }^{2}$.

É perceptível que apesar dos partos por via vaginal serem predominantes, os partos por via cesárea vêm crescendo ao longo dos anos estudado. Segundo a Organização Mundial de Saúde (OMS), o índice razoável de cesáreas é de
$15 \%$ dos nascimentos. No Brasil, $55,6 \%$ dos 2,9 milhões de partos realizados anualmente são cirúrgicos. A ANS realiza, há mais de uma década, um trabalho contínuo para a promoção do parto normal e a redução do número de cesarianas desnecessárias na saúde suplementar, que, atualmente, atinge alarmantes índices ${ }^{20}$.

Verificou-se, no presente estudo, um crescente número de óbitos entre mães, com faixa etária entre 20 e 29 anos, autodenominadas pardas, com 8 a 11 anos de estudos. Fonseca et al. $^{21}$ (2017), em seu estudo, diz que, existe o efeito combinado entre a idade materna e a baixa escolaridade aumentam a chance de óbito neonatal, devido a se tornarem grupos vulneráveis devido a um baixo nível de conhecimento.

A literatura ressalta ainda que, é possível que a situação socioeconômica da mãe reflita no acesso as diferentes condições de atenção à saúde, condição importante para a identificação de grupos populacionais para os quais devem ser priorizados os cuidados de saúde, particularmente durante a gravidez, o parto e o período neonatal ${ }^{22}$. Visto que, é essencial, e de grande importância o acesso das gestantes aos serviços de saúde, de forma a garantir um acompanhamento adequado, com realização do pré-natal, no intuito de evitar intercorrências.

De acordo com o Manual dos comitês de prevenção do óbito infantil e fetal, de 2004, do Ministério da Saúde, a maior parte dos óbitos neonatais ocorrem no período neonatal precoce (0-6 dias de vida), cerca de $40 \%$ no primeiro dia de vida, e um número significativo acontece ainda nas primeiras horas de vida, evidenciando a estreita relação entre os óbitos infantis e a assistência de saúde nas maternidades. Além disso, a mortalidade neonatal precoce pode estar subestimada pelo registro de óbitos declarados como natimortos, que de fato são óbitos de nascidos vivos ocorridos pouco após o parto ${ }^{23}$, o que pode repercutir nos índices reais dos dados deste estudo.

Observa-se com as informações do presente estudo que o óbito neonatal permanece elevado, e quando analisados isoladamente, comparando as taxas do ano de maior concentração, deste estudo, o período neonatal precoce $(10,24$ para cada 1.000 nascidos vivos), e tardio (2,85 para cada 1.000 nascidos vivos), ambos no ano de 2013, nota-se que o precoce apresenta uma ocorrência quase quatro vezes maior comparado ao tardio, confirmando o que o Manual dos comitês de prevenção do óbito 
infantil e fetal do Ministério da Saúde descreve que a maior ocorrência de mortalidade infantil se situa como neonatal precoce ${ }^{23}$.

Taxas elevadas em geral estão vinculadas as causas preveníveis, relacionadas as condições insatisfatórias em relação ao nível socioeconômico da população, ao acesso e à utilização dos serviços de saúde, como à saúde da mãe, bem como à inadequada assistência prénatal, ao parto e ao recém-nascido ${ }^{23}$.

O período neonatal é uma fase considerada de vulnerabilidade à saúde infantil por riscos biológicos, ambientais, sociais e culturais. Isso requer cuidados adequados, uma maior vigilância e acompanhamento por parte do profissional de saúde, a fim de garantir um melhor crescimento e desenvolvimento da criança $^{24}$.

Segundo Aguiar \& Cols $^{25}$, existem alguns desafios para o funcionamento adequado do SUS, a destacar, as desigualdades socioeconômicas, à heterogeneidade das estruturas e instituições de saúde e às condições epidemiológicas que caracterizam as diversas regiões e municípios.

Para Pinheiro et al. $^{26}$, as políticas vigentes de proteção à saúde da criança tentam priorizar as famílias mais vulneráveis. Os programas e as estratégias tentam fortalecer e qualificar o serviço público para desenvolver essas ações, das quais depende a maior parte da população brasileira.

O Brasil tem firmado compromissos internos e externos para a melhoria da qualidade da atenção à saúde prestada à gestante e ao recém-nascido, com o objetivo de reduzir a mortalidade materna e infantil. Às estratégias implementadas pelo governo federal, como ações para diminuição da pobreza, ampliação da cobertura da Estratégia Saúde da Família, entre outras $^{16}$.

Neste sentido, o Ministério da Saúde, organizou uma grande estratégia, a fim de qualificar as Redes de Atenção Materno-Infantil em todo país, com vistas à redução das taxas, ainda elevadas, de morbimortalidade materna e infantil. Trata-se da Rede Cegonha, que foi uma proposta implementada através da Portaria $n$ o 1.459/GM/MS, de 24 de junho de 2011, com o intuito de criar iniciativas que envolvem mudanças no modelo de cuidado à gravidez, ao parto/nascimento e a atenção integral à saúde da criança, com foco nos primeiros dois anos e, em especial no período neonatal. Além disso, propõe garantir a todos os recém-nascidos boas práticas de atenção, embasadas em evidências científicas e nos princípios de humanização ${ }^{16}$.
Em dezembro de 2014, de acordo com os dados das Tábuas Completas de Mortalidade divulgadas pelo Instituto Brasileiro de Geografia e Estatística (IBGE), a taxa de mortalidade infantil ficou em 15 bebês para cada mil nascidos vivos em 2013, considerando crianças de até 1 ano de idade. Em 2012, a proporção foi de 15,7 óbitos na mesma faixa etária para cada mil nascidos vivos. De 1990 a 2012, a taxa de mortalidade infantil no Brasil reduziu $70,5 \%{ }^{27}$.

Foi observado uma inconsistência/ou incompletude de algumas das variáveis citados como "Ignorado", que apresentam em proporções consideráveis no presente estudo, no qual, destacou-se, como exemplo nas variáveis: escolaridade e idade da mãe, peso ao nascer e raça/cor do RN. Esta incompletude, de certa forma, interfere na qualidade dos dados, e na precisão dos resultados, visto que, são de extrema importância e trazem maior veracidade as informações apresentadas, quando estes, estão em sua totalidade. No qual, cabe, aos profissionais de saúde manterem uma comunicação efetiva, que estabeleça uma transparência entre o usuário, a fim de, mapear os indicadores de forma real.

De acordo com a Versão preliminar do Roteiro de análise da qualidade da base de dados e cálculo de indicadores epidemiológicos e operacionais, uma base de dados de boa qualidade deve ser completa, fidedigna aos dados originais registrados nas unidades de saúde, transpassando confiabilidade, sem duplicidades e seus campos devem estar preenchidos de forma consistentes ${ }^{28}$.

\section{Considerações finais}

O presente estudo permitiu explanar, os principais fatores que estão possivelmente associados ao óbito neonatal na Região de Saúde de Salvador, Bahia. No qual observou que os óbitos neonatais, em geral estão vinculadas as condições sociodemográficas, associados a fatores socioeconômicos da mãe e/ou família, além de serem oriundos de questões assistenciais tanto no período do pré-natal quanto no parto, o que se deve considerar como um alerta para os serviços de saúde.

Dessa forma, com o conhecimento dos resultados dessa investigação conclui-se que, a Atenção Primária à saúde possui uma estrutura eficaz para a redução dos óbitos infantis, porém, precisa de um método de ação, ou seja, uma estratégia de evitabilidade competente pelos serviços de saúde a fim de que à prevenção de acordo com os fatores identificados neste 
estudo, sejam utilizados como forma de monitorar e diminuir as taxas de óbitos.

Além disso, vale ressaltar o estímulo a utilização de práticas humanizadas nos serviços, educação em saúde pelos profissionais em comunidades, facilitando a acessibilidade das mães/gestantes, em que reconheçam a importância sobre a busca pelos sistemas de saúde precoce, além de comparecer e usufruir dos programas propostos pela Atenção primária a saúde, a exemplo do pré-natal que seja desenvolvido com uma atenção com qualidade assegurada, na perspectiva de minimizar consequências para a saúde das mães e dos bebês.

\section{Referências}

1. Brasil. Indicadores básicos para a saúde no Brasil: conceitos e aplicações / Rede Interagencial de Informação para a Saúde - Ripsa. - 2. ed. - Brasília: Organização Pan-Americana da Saúde, 2008. 349 p.: il.

2. Franca EB, Lansky S. Mortalidade Infantil Neonatal no Brasil: Situação, Tendências e Perspectivas. In: Rede Interagencial para Saúde, organizador. Demografia e saúde: contribuição para análise de situação e tendências, Brasília, 2009.

3. Brasil. Secretaria do Estado de Saúde. Diminui o índice de mortalidade infantil no Brasil. São Paulo, 2016. [Acesso em 10 abr. 2020] Disponível em: http://www.saude.sp.gov.br/instituto-desaude/transparencia/ultimas-noticias/diminui-oindice-de-mortalidade-infantil-no-brasil.

4. Almeida SD, Barros MBA. Atenção à saúde e mortalidade neonatal: estudo casocontrole realizado em Campinas, SP. Revista Brasileira de Epidemiologia. São Paulo, 2004; 7(1).

5. Lansky S, Friche AAL, Silva AAM, Campos D, Bittencourt DAS, Carvalho ML, Frias PG, Cavalcante RS, Cunha AJL. Pesquisa Nascer no Brasil: perfil da mortalidade neonatal e avaliação da assistência à gestante e ao recém-nascido. Cad. Saúde Pública. Rio de Janeiro, 2014; 30(1).

6. Teixeira JAM, Araujo WRM, Maranhao AGK, Escalante JJC, Rezende LFM, Matijaevich A. Mortalidade no primeiro dia de vida: tendências, causas de óbito e evitabilidade em oito Unidades da Federação brasileira, entre 2010 e 2015. Rev. Bras. Epidemiologia. São Paulo, 2019, Fevereiro; 18(1).

7. Dias RA. A Importância do Pré-natal na Atenção Básica. Minas Gerais, 2014. [Acesso em 11 abr. 2019] Disponível em: https://www.nescon.medicina.ufmg.br/bibliotec a/imagem/Importancia_pre_natal_aten\%C3\%A7 ao_basica.pdf.

8. Brasil. Secretaria do Estado de Saúde. Atenção Básica ou Primária - Principal porta de entrada para o Sistema Único de Saúde (SUS). Porto Alegre, 2019. [Acesso em 10 abr. 2020] Disponível em: https://saude.rs.gov.br/atencaobasica-ou-primaria-principal-porta-de-entradapara-o-sistema-unico-de-saude-sus.

9. Brasil. Ministério da Saúde. Secretaria de atenção à Saúde. Departamento de atenção básica. Atenção ao pré-natal de baixo risco. Brasília: Ministério da Saúde, p. 316, 2012.

10. Silva CF, Leite AJM, Almeida NMG, Leonlbironke ACMP. Fatores associados ao óbito neonatal de recém-nascidos de alto risco: estudo multicêntrico em Unidades Neonatais de Alto Risco no Nordeste brasileiro. Cad. Saúde Pública. Rio de Janeiro, 2014, Fev.; 30(2).

11. Luz LA, Aquino R, Medina MG. Avaliação da qualidade da Atenção Pré-Natal no Brasil. Saúde debate. Rio de Janeiro, Oct. 2018; 42(2). [Acesso em 13 mai. 2021]. Disponível em:https://www.scielo.br/scielo.php?script=sci arttext\&pid=S010311042018000600111\#: :text= A\%20Aten\%C3\%A7\%C3\%A30\%20Pr\%C3\%A9\%2D Natal\%20(APN,padr\%C3\%B5es\%20t\%C3\%A9cnic o\%2Dcient\%C3\%ADficos\%20de\%20qualidade.

12. Gavaia MAM, Fujimori E, Sato APS. Mortalidade neonatal: análise das causas evitáveis. Rev. Enferm. UERJ. Rio de Janeiro, 2015, Mar-Abr; 23(2), p. 247-253.

13. IBGE. Estatísticas de Salvador, 2019. [Acesso em 28 mar. 2020]. Disponível em: https://www.ibge.gov.br/cidades-eestados/ba/salvador.html?.

14. Baltrusis N, Mourad LN. Política habitacional e locação social em Salvador. Cad. CRH. Salvador, 2014, Junho- Agosto; 27(71).

15. EGESTOR. Ministério da Saúde. Informação e Gestão de Atenção Básica. [Acesso em 13 mai. 2021]. Disponível em: https://egestorab.saude.gov.br/paginas/acessoP ublico/relatorios/relHistoricoCobertura.xhtml.

16. Brasil. Ministério da Saúde. Secretaria de Atenção à Saúde. Departamento de Ações Programáticas Estratégicas. Atenção à saúde do recém-nascido: guia para os profissionais de saúde - Cuidados Gerais. - 2. ed. atual. Brasília: Ministério da Saúde, 2014.

17. Bezerra NF, Rassy MEC, Alves BLA, Carvalho TCN, Bandeira FJS. Fatores relacionados à mortalidade neonatal. Rev. Enferm. UFPE online. Recife, 2016, Nov.; 10(11).

18. Araújo BF, Bozzeti MC, Tanaka AC. Mortalidade neonatal precoce no município de 
Caxias do Sul: um estudo de coorte. Jornal de Pediatria. Caxias do Sul, 2000; 76(3).

19. Granzotto JÁ, Fonseca SS, Lindermann FL. Fatores relacionados à mortalidade neonatal em uma unidade de terapia intensiva neonatal na região sul do Brasil. Rev. AMRIGS [Internet]. Jan/Mar. 2012; 56(1), p. 57-62.

20. Brasil. Agência Nacional de Saúde Suplementar. Parto é Normal. Rio de Janeiro. [Acesso em 18 mai. 2021] Disponível em: http://www.ans.gov.br/parto-e-normal.

21. Fonseca SC, Flores PVG, Camargo Jr KR, Pinheiro RS, Coeli CM. Escolaridade e idade materna: desigualdades no óbito neonatal. Rev Saude Publica. 2017; p.51:94.

22. Soares ES, Menezes GMS. Fatores associados à mortalidade neonatal precoce: análise de situação no nível local. Revista de Epidemiol. Serv. Saúde. Salvador, 2010; 19(1).

23. Brasil. Ministério da Saúde. Secretaria de Atenção à Saúde. Departamento de Ações Programáticas Estratégicas. Manual dos comitês de prevenção do óbito infantil e fetal / Ministério da Saúde, Secretaria de Atenção à Saúde, Departamento de Ações Programáticas Estratégicas. - Brasília: Ministério da Saúde, 2004.
24. Brasil. Ministério da Saúde (MS). Secretaria de Atenção à Saúde. Departamento de Ações Programáticas e Estratégicas. Atenção à saúde do recém-nascido: guia para os profissionais de saúde. Brasília: Ministério da Saúde (MS). 2011; v.1.

25. Aguiar ZN (orgs.). SUS: Sistema Único de Saúde - Antecedentes, Percursos, Perspectivas e Desafios. 1a. ed. São Paulo: Editora Martinari, 2011.

26. Pinheiro JMF, Tinoco LS, Rocha ASS, Rodrigues MP, Lyra CO, Ferreira MAF. Atenção à criança no período neonatal: avaliação do pacto de redução da mortalidade neonatal no Rio Grande do Norte, Brasil. Ciênc. saúde colet. Jan, 2016; 21(1), p.243-252.

27. Brasil. Ministério da Saúde. Rede Cegonha | Brasil apresenta queda nos índices de mortalidade infantil. Dezembro de 2014. [Acesso em 18 mai. 2021] Disponível em: http://www.blog.saude.gov.br/34876-redecegonha-brasil-apresenta-queda-nos-indices-demortalidade-infantil.

28. BRASIL. Ministério da Saúde. Roteiro para uso do SINAN net, análise da qualidade da base de dados e cálculo de indicadores epidemiológicos e operacionais (Versão Preliminar) - Brasília - DF; 2019.

\section{Endereço para Correspondência}

Maísa Mônica Flores Martins

Avenida Pinto de Aguiar, 2589 - Pituaçu, Salvador

$-B A$

CEP: $41740-090$

E-mail: maisamonica@gmail.com

Recebido em 29/01/2021

Aprovado em 24/05/2021

Publicado em 15/06/2021 